OPEN ACCESS

Edited by:

Volker Mall,

Technical University of

Munich, Germany

Reviewed by:

Patricia Rzezak,

University of São Paulo, Brazil

Lino Nobili,

University of Genoa, Italy

Chao-Ying Chen,

Hong Kong Polytechnic

University, China

${ }^{*}$ Correspondence:

Alisa Gschaidmeier

agschaidmeier@schoen-klinik.de

Specialty section:

This article was submitted to

Pediatric Neurology,

a section of the journal

Frontiers in Pediatrics

Received: 28 January 2021

Accepted: 06 May 2021

Published: 31 May 2021

Citation:

Gschaidmeier A, Heimgärtner $M$, Schnaufer L, Hernáiz Driever P. Wilke M, Lidzba K and Staudt M (2021) Non-verbal Intelligence in Unilateral Perinatal Stroke Patients

With and Without Epilepsies.

Front. Pediatr. 9:660096.

doi: 10.3389/fped.2021.660096

\section{Non-verbal Intelligence in Unilateral Perinatal Stroke Patients With and Without Epilepsies}

\author{
Alisa Gschaidmeier ${ }^{1,2 *}$, Magdalena Heimgärtner ${ }^{1}$, Lukas Schnaufer ${ }^{1,3}$, \\ Pablo Hernáiz Driever ${ }^{4}$, Marko Wilke ${ }^{1,3}$, Karen Lidzba ${ }^{1,5}$ and Martin Staudt ${ }^{1,2}$ \\ ${ }^{1}$ Department of Pediatric Neurology and Developmental Medicine, University Children's Hospital, Tübingen, Germany, \\ ${ }^{2}$ Center for Pediatric Neurology, Neurorehabilitation and Epileptology, Schön Klinik Vogtareuth, Vogtareuth, Germany, \\ ${ }^{3}$ Experimental Pediatric Neuroimaging, Children's Hospital and Department of Neuroradiology, University Hospital, Tübingen, \\ Germany, ${ }^{4}$ Department of Pediatric Oncology and Hematology, Berlin Institute of Health, Charité-Universitätsmedizin Berlin, \\ Freie Universität Berlin, Humboldt-Universität zu Berlin, Berlin, Germany, ${ }^{5}$ Division of Neuropediatrics, Development, and \\ Rehabilitation, University Children's Hospital Inselspital, Bern University Hospital, University of Bern, Bern, Switzerland
}

Background: The risk factors for impaired cognitive development after unilateral perinatal stroke are poorly understood. Non-verbal intelligence seems to be at particular risk, since language can shift to the right hemisphere and may thereby reduce the capacity of the right hemisphere for its originary functions. Pharmaco-refractory epilepsies, a frequent complication of perinatal strokes, often lead to impaired intelligence. Yet, the role of well-controlled epilepsies is less well-understood. Here, we investigated whether well-controlled epilepsies, motor impairment, lesion size, lesion side, and lateralization of language functions influence non-verbal functions.

Methods: We recruited 8 patients with well-controlled epilepsies (9-26 years), 15 patients without epilepsies (8-23 years), and 23 healthy controls (8-27 years). All underwent the Test of Non-verbal Intelligence, a motor-independent test, which excludes biased results due to motor impairment. Language lateralization was determined with functional MRI, lesion size with MRI-based volumetry, and hand motor impairment with the Jebson-Taylor Hand Function-Test.

Results: Patients with epilepsies showed significantly impaired non-verbal intelligence $[\mathrm{Md}=89.5$, interquartile range $(\mathrm{IQR})=13.5]$ compared with controls $(\mathrm{Md}=103$, $I Q R=17)$. In contrast, patients without epilepsies ( $M d=97, I Q R=15.0)$ performed within the range of typically developing children. A multiple regression analysis revealed only epilepsy as a significant risk factor for impaired non-verbal functions.

Conclusion: In patients with unilateral perinatal strokes without epilepsies, the neuroplastic potential of one healthy hemisphere is able to support the development of normal non-verbal cognitive abilities, regardless of lesion size, lesion side, or language lateralization. In contrast, epilepsy substantially reduces this neuroplastic potential; even seizure-free patients exhibit below-average non-verbal cognitive functions.

Keywords: early brain lesion, functional magnet resonance imaging, lesion size, motor impairment, cognitive function 


\section{INTRODUCTION}

Perinatal stroke has an estimated birth-prevalence of 37$64 / 100,000(1,2)$. It affects mostly term-born newborns, and presents with diverse signs and symptoms (2). Outcome is quite variable, with many children achieving normal levels of function $(3,4)$, while others experience difficulties, such as impairment in different domains of cognitive functions (5-7) as well as in motor skills (8-10). Potentially modifying factors explaining the variability in cognitive outcome are lesion size, the severity of hand motor impairment (6), and the side of the lesion (11-13).

In addition, for non-verbal functions in patients with leftsided lesions, language reorganization has been proposed as a modifying factor $(6,7,14)$. This has been explained in the context of the "crowding hypothesis" (15-17), suggesting that cognitive processes originally located in the right hemisphere such as nonverbal intelligence show deficits when language functions shift to the right hemisphere. Following this hypothesis, non-verbal functions seem to be at a particular risk for lower performance; hence, in this study, we chose to focus on the development of non-verbal functions after perinatal stroke.

The threat most feared by families of children with perinatal stroke, however, is the development of epilepsy. Indeed, patients with perinatal stroke have a significant risk to develop epilepsy, which was estimated between $15 \%$ (18) and 54\% (19). Not surprisingly, the type of the lesion also plays an important role, since children with cortico-subcortical lesions (commonly due to arterial ischemic strokes, AIS), are much more prone to develop epilepsies than children with white matter lesions (usually due to periventricular venous infarctions, PVI) $(7,20)$. In addition to the burden of seizures and side effects of anti-epileptic medication, epilepsy may also hamper cognitive development. For children with pharmaco-refractory epilepsies due to perinatal strokes, it is well-known that cognitive development can be severely compromised $(3,4,21,22)$. Much less is known, whether less severe, well-controlled epilepsies also play a role in this regard.

A frequent methodological problem in almost all previous studies on non-verbal cognitive abilities after perinatal stroke was the application of tests requiring bimanual activities, like the Wechsler Intelligence Scales (3, 4, 23-25), the Kaufman Assessment Battery for Children (7), or the Beery Developmental Test of Visual-Motor Integration (4). Administering such tests in hemiparetic patients - a frequent consequence of perinatal stroke $(9,10)$ - can lead to artificially lower scores for nonverbal functions [see (26) for review]. To overcome this problem, some authors [e.g., (6)] simply excluded patients with substantial motor impairments that prevented valid administration of the measures. As this potentially biases the outcomes, we here opted to use a different approach: the assessment of non-verbal cognitive abilities with completely motor-free tests. In a previous study (14), we had used the Block Tapping test and the Tube Figures Test; in the current study, we used the Test Of Nonverbal Intelligence (TONI-4) which measures the ability for abstract reasoning and the problem-solving capability-without involving any motor component $(27,28)$.

Motor impairment may therefore influence the result of cognitive tests by different mechanism. First, as described above, motor impairment can artificially influence the test procedure itself. Second, impaired motor abilities might limit a child's abilities to explore its environment, and thereby impair also the development of cognitive functions (29).

The aim of our study therefore was to assess the influence of well-controlled epilepsy (which we arbitrarily defined as seizure-freedom for at least 6 months) on non-verbal cognitive development of children with perinatal strokes, as assessed using appropriate test procedures. Furthermore, we wanted to clarify the influence of lesion size, hand motor impairment, side of the lesion, and language lateralization on non-verbal cognitive performances.

\section{METHOD}

\section{Subjects}

Participants were recruited in the University Children's Hospital Tübingen and in the Center for Pediatric Neurology, Neurorehabilitation and Epileptology, Schön Klinik Vogtareuth.

In Tübingen, participants were recruited by searching the clinical database for relevant diagnoses in electronic patient charts and by personal contacts to patients who had participated in previous studies. Healthy controls were recruited via advertisements in the local press and in the clinic internal information system. Patients in Vogtareuth were recruited via personal invitations after searching the clinical database and during hospitalization for a motor skills rehabilitation training. These differences in recruitment strategies may explain why the two cohorts of patients differed in terms of age (median age Tübingen $=18.13$ years, median age Vogtareuth $=10.15$ years). Inclusion criteria, however, were the same, and all participants were included following telephone interviews with identical questionnaires.

We included 23 patients (11 females; age range 8-26 years; median age 12.56 years) with a diagnosis of a pre-, peri-, or neonatally acquired unilateral arterial ischemic stroke (AIS) or unilateral periventricular hemorrhagic infarction (PVI). In order to be able to participate in all tests, patients had to be native German-speaking and aged 8 years or older. Patients with a previous diagnosis of intellectual disability (defined as IQ below 70) were excluded. Controls were screened using a questionnaire asking for any neurological or psychiatric diagnosis and for problems in cognitive or language development. We did not, however, use any formal assessment confirming normal development.

Additionally, patients with seizures during the last 6 months were excluded. Hemiparesis was present in 21/23 patients (no hemiparesis in \#V13, \#T12).

Patients were diagnosed with epilepsy $(n=8$; age range, 9-26 years; median age, $16.40 ; 2$ females) when at least two afebrile, unprovoked seizures had occurred in the post-neonatal period [definition as suggested in $(19,30)$ ]. All but one of these patients (\#T28) were on anti-convulsive medication at the time of the study. Of the patients without epilepsies $(n=15$; age range, 8-23 years; median age, 11.80; 9 females), one patient (\#V03) had suffered one single seizure, but was never treated with anti-convulsive medication. The other 14 patients never 
had experienced epileptic seizures. Twenty-three age-matched healthy volunteers (age range 8-27 years; median age, 12.42 years; 8 females) served as controls. For every single patient, we agematched a person from our cohort of 38 controls-irrespective of epilepsy.

The study was approved by the local ethics committee (Nr. 693/2014B01). All adult participants and the parents of underage participants gave their written, informed consent, and all underage participants gave verbal assent. The study was in accordance with the Code of Ethics of the World Medical Association (Declaration of Helsinki, 1964 in its latest version).

\section{Neuropsychological Assessment}

All participants completed the Test of Non-verbal Intelligence, Fourth Edition (TONI-4), which measures the ability for abstract reasoning and the problem-solving capability (28). It contains of 60 graphical items, arranged in order from easy to difficult. The participants analyze similarities and differences between the items, which are defined by the characteristics shape, position, direction, rotation, contiguity, shading, size and movement. The age-adjusted test results are not confounded by motor deficits and are therefore suitable for patients with motor impairments such as hemiparesis (27).

\section{Structural and Functional Magnetic Resonance Imaging}

Subjects were either scanned on a Siemens $1.5 \mathrm{~T}$ Avanto (Tübingen) or Symphony (Vogtareuth) MRI scanner (Siemens Medizintechnik, Erlangen, Germany), using the same sequences and a standard quadrature head coil. An echo-planar imaging (EPI) sequence was used to acquire functional series (repetition time $=3,000 \mathrm{~ms}$, echo time $=40 \mathrm{~ms}, 40$ axial slices, in plane matrix $=64 \times 64$, covering the whole brain with a voxel size $=3$ $\times 3 \times 3 \mathrm{~mm}^{3}$ ). Anatomical images were acquired as T1-weighted $3 \mathrm{D}$-datasets (repetition time $=1,300 \mathrm{~ms}$, echo time $=2.92 \mathrm{~ms}$, 167 contiguous sagittal slices, in plane matrix $265 \times 265$, resulting in a voxel size of $1 \times 1 \times 1 \mathrm{~mm}^{3}$ ).

Functional and anatomical images were pre-processed and analyzed using SPM12 (Statistical Parametric Mapping; Wellcome Department of Imaging Neurosciences, UCL, UK), the Computational Anatomy Toolbox extension to SPM12 (CAT12, by Christian Gaser and Robert Dahnke, Departments of Psychiatry and Neurology, Jena University Hospital), as well as custom scripts and functions running within Matlab (Mathworks, Natrick MA, USA).

For the determination of lesion size, individual lesion masks were manually drawn in native space on the anatomical T1-weighted image using MRIcron (31). To compensate for asymmetric ventricular enlargement, the mirrored ventricles of the contra-lesional hemisphere were excluded from the lesion masks for all subjects. Lesion volumes were calculated from the lesion masks using a custom script.

For the determination of language lateralization, all patients with left-sided lesions underwent fMRI and performed the Vowel Identification Task (32) as a word generation task. Methodological details have been described elsewhere (32-34). In brief, pictures of everyday objects were presented visually to the participants, who were asked to decide if the name of the object contained the phoneme $<\mathrm{i}>$ by silently generating the name of the object. In the control condition, participants were presented an abstract puzzle and were asked to decide if a small piece fitted into a larger one. Laterality of language activation was calculated as described in Lidzba et al. (35). Based on the resulting laterality index (LI), patients were classified as "left-dominant" (LI $>+0.2)$, as "right-dominant" $(\mathrm{LI}<-0.2)$ or as "bilateral" $(-0.2 \leq \mathrm{LI} \leq+0.2)$.

\section{Motor Assessment}

Hand motor function was assessed with the Jebson Taylor Hand function test (JTHFT). The test provides quantitative measurements of standardized unimanual hand function tasks that are frequently used in everyday activities (36). We calculated the median of six subtests (card turning, picking up small objects, stacking checkers, simulated feeding, lifting light objects and lifting heavy objects) (37). As suggested previously, we did not perform the subtest "writing" due to possible distortion by the different ages and performance levels of the participants (3840). The test was initially developed for adults, but recently showed a good test-retest reliability in children aged 6-10 years (41). For our analysis, we calculated the ratio of the medians "non-dominant"/ "dominant" hand motor function to control for inter-individual differences not related to the hemiparesis.

\section{Statistics}

The statistical analyzes were performed using SPSS 25. For correlation analyses, we used Spearman rank correlations; for correlations including dichotomous variables, we used the pointbiserial correlations. Significance was assumed at $p \leq 0.05$, For group comparisons between the three groups, we used the non-parametric Kruskal-Wallis-test, corrected for multiple comparisons where appropriate by Bonferroni correction. A ChiSquare-test was used for a distribution measurement of the control group. Multiple regression analyses were used for the assessment of several predictor variables for the outcome variable non-verbal intelligence.

\section{RESULTS}

Table 1 summarizes demographic and clinical data of all 23 patients. Lesion size could be calculated in $21 / 23$ patients, ranging from 1.16 to $220.71 \mathrm{~cm}^{3}$. In the other two, dental braces (\#V04) or an implanted shunt system (\#T28) prevented the reliable determination of lesion size (Table 1). The presence of epilepsy correlated significantly with lesion size (point-biserial correlation, $r=-0.47, p<0.01$ ) and with the type of the lesion (AIS vs. PVI, Phi $p<0.05$ ).

The Kruskal Wallis-test for the three groups-patients with epilepsy, patients without epilepsy, and controls-yielded a significant main effect for non-verbal intelligence $[\mathrm{H}(2)=9.06$, $n=46, p<0.05]$. Pairwise comparisons with Bonferroniadjusted $p$-values demonstrated that patients with epilepsy scored significantly lower than controls $(p=0.010)$, while no significant differences were observed between patients without epilepsy and 
TABLE 1 | Patient characteristics.

\begin{tabular}{|c|c|c|c|c|c|c|c|c|c|c|c|c|c|c|}
\hline ID & $\begin{array}{c}\text { Age at } \\
\text { study } \\
\text { (years) }\end{array}$ & Sex & Lesion type & TONI & Epilepsy & $\begin{array}{l}\text { Lesion } \\
\text { size } \\
(\mathrm{ml})\end{array}$ & $\begin{array}{l}\text { JTHFT } \\
\text { (ratio) }\end{array}$ & Lesion side & LI & $\begin{array}{c}\text { Age of } \\
\text { onset } \\
\text { (years) }\end{array}$ & $\begin{array}{l}\text { Time since } \\
\text { seizure- } \\
\text { freedom } \\
\text { (years) }\end{array}$ & $\begin{array}{l}\text { History of } \\
\text { status } \\
\text { epilepticus }\end{array}$ & $\begin{array}{l}\text { History of } \\
\text { AEDs }\end{array}$ & $\begin{array}{l}\text { Time between } \\
\text { first and } \\
\text { last seizure } \\
\text { (years) }\end{array}$ \\
\hline T14 & 16 & M & AIS & 77 & Yes & 133.93 & 1.37 & Left & -0.5 & 11 & 1.25 & No & $\begin{array}{l}\text { LEV BRIV, } \\
\text { OXC }\end{array}$ & 4 \\
\hline T39 & 26 & M & AIS & 87 & Yes & 167.79 & 25.34 & Left & -0.87 & 11.5 & 8 & No & STM & 1.5 \\
\hline T44 & 16 & M & AlS & 87 & Yes & 84.97 & 9.48 & Right & & $\mathrm{N} / \mathrm{A}$ & $\mathrm{N} / \mathrm{A}$ & $\mathrm{N} / \mathrm{A}$ & OXC & \\
\hline $\mathrm{T} 41$ & 22 & M & $\mathrm{PVI}$ & 89 & Yes & 200.57 & 6.49 & Right & & 15.5 & 6 & No & OXC & 1 \\
\hline V11 & 10 & $M$ & PVI & 90 & No & 50.29 & 4.69 & Left & +0.63 & & & & & \\
\hline T28 & 16 & M & Not classified & 90 & Yes & $\mathrm{N} / \mathrm{A}$ & 1.52 & Left & +0.69 & 4 & 1 & Yes & $\begin{array}{l}\text { STM, LEV, } \\
\text { CLB, PHT }\end{array}$ & 12 \\
\hline V04 & 15 & $\mathrm{~F}$ & $\mathrm{PVI}$ & 91 & No & $\mathrm{N} / \mathrm{A}$ & 5.75 & Right & & & & & & \\
\hline $\mathrm{T} 21$ & 23 & $\mathrm{~F}$ & $\mathrm{PVI}$ & 92 & No & 29.57 & 6.64 & Left & -0.25 & & & & & \\
\hline V14 & 10 & M & PVI & 93 & No & 79.38 & 18.65 & Right & & & & & & \\
\hline V06 & 8 & M & $\mathrm{PVI}$ & 93 & No & 28.83 & 2.40 & Left & +0.78 & & & & & \\
\hline T57 & 19 & $\mathrm{~F}$ & $\mathrm{PVI}$ & 93 & No & 1.16 & 1.97 & Left & +0.64 & & & & & \\
\hline T33 & 23 & M & $\mathrm{PVI}$ & 97 & No & 161.77 & 4.08 & Left & -0.66 & & & & & \\
\hline V03 & 11 & $\mathrm{~F}$ & PVI & 97 & No & 55.82 & 2.94 & Left & +0.67 & & & & & \\
\hline T19 & 23 & $\mathrm{~F}$ & $\mathrm{PVI}$ & 99 & No & 36.05 & 2.42 & Left & -0.83 & & & & & \\
\hline $\mathrm{T} 13$ & 9 & $M$ & $\mathrm{PVI}$ & 99 & Yes & 22.82 & 1.58 & Left & +0.85 & 0.25 & 0.5 & Yes & $\begin{array}{l}\text { LEV, ACTH, } \\
\text { PB, STM, } \\
\text { CLB, } \\
\text { Steroids, } \\
\text { ESM }\end{array}$ & 8 \\
\hline V01 & 8 & $M$ & PVI & 100 & No & 24.80 & 15.25 & Left & +0.61 & & & & & \\
\hline V12 & 10 & $\mathrm{~F}$ & $\mathrm{PVI}$ & 101 & Yes & 220.71 & 5.05 & Right & & 1 & 3 & No & $\begin{array}{l}\text { OXC, TPM, } \\
\text { LEV }\end{array}$ & 6 \\
\hline V13 & 10 & $\mathrm{~F}$ & PVI & 102 & No & 85.36 & 0.95 & Left & -0.31 & & & & & \\
\hline V02 & 8 & $\mathrm{~F}$ & $\mathrm{PVI}$ & 104 & Yes & 45.11 & 2.04 & Right & & 4.5 & 3 & No & LEV & 1.5 \\
\hline V09 & 10 & $\mathrm{~F}$ & PVI & 108 & No & 1.24 & 2.17 & Left & N/A & & & & & \\
\hline T12 & 12 & $\mathrm{~F}$ & $\mathrm{PVI}$ & 109 & No & 97.30 & 1.12 & Left & -0.32 & & & & & \\
\hline T56 & 12 & $M$ & PVI & 109 & No & 9.08 & 2.39 & Right & & & & & & \\
\hline V08 & 11 & $\mathrm{~F}$ & AIS & 121 & No & 41.65 & 3.85 & Left & -0.53 & & & & & \\
\hline
\end{tabular}

Patients are sorted by TONI. Hand motor function is calculated by the ratio (non-dominant/dominant) hand function of the median scores of JTHFT.

AIS, arterial ischemic stroke; LI, laterality index (positive: left; negative: right); N/A, no data available due to incompliance or technical failure; PVI, periventricular venous infarction; JTHFT, Jebson Taylor Hand function test; TONI, Test of non-verbal intelligence. LEV, Levetiracetam; BRV, Brivaracetam; OXC, Oxcarbazepine; STM, Sulthiame; CLB, Clobazam; PHT, Phenytoin; PB, Phenobarbital; ESM, Ethosuximide; TPM, Topiramate.

non-verbal intelligence. LEV, Levetiracetam; BRV, Brivaracetam; OXC, Oxcarbazepine; STM, Sulthiame; CLB, Clobazam; PHT, Phenytoin; PB, Phenobarbiation
${ }^{*} T$ he lesion in T28 could not unequivocally be classified as AIS or PVI because only MRI after implantation of a ventriculoperitoneal shunt were available. 


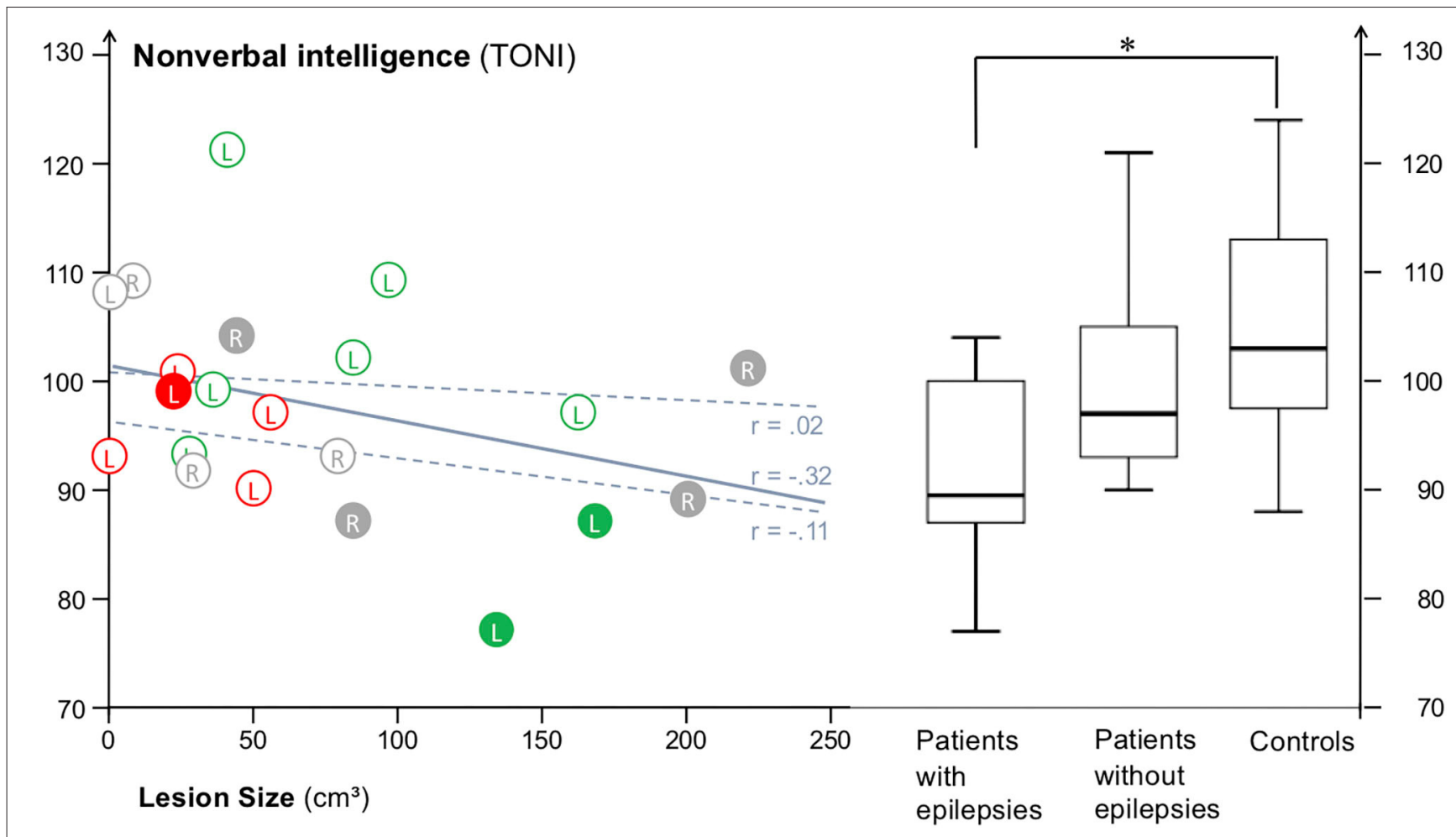

FIGURE 1 | Scatterplot of all 21 patients with lesion size data available, marked with solid circles (with epilepsy) or open circles (without epilepsy). Capital letters mark the side of the lesion ( $L, R$ ), colors mark the lateralization of language (red = left-dominant; green = right-dominant; gray = no information). The solid line indicates the correlation between lesion size and TONI for all patients, the dashed lines the correlations for the subgroups of patients with epilepsies (lower line) and without epilepsies (upper line). The median TONI scores were about 7.5 points lower for patients with epilepsies $(\mathrm{Md}=89.5, \mathrm{SD}=9.0)$ vs. those without epilepsies (Md =97, $\mathrm{SD}=8.8$ ), and almost one standard deviation lower for patients with epilepsy vs. typically developing controls (Md $=103, \mathrm{SD}=10.0)$. Patients with epilepsy differed significantly (Kruskal-Wallis-test) from controls (marked with *), while patients with and without epilepsy and patients without epilepsy and controls did not differ.

TABLE 2 | Correlation coefficients.

\begin{tabular}{|c|c|c|c|c|c|}
\hline & Epilepsy & $\begin{array}{l}\text { JTHFT } \\
\text { (ratio) }\end{array}$ & Lesion size & Lesion side & $\begin{array}{c}\text { LI } \\
\text { (left-sided lesions only) }\end{array}$ \\
\hline TONI-4 & $-0.405^{\star}$ & $-0.359^{\star}$ & -0.324 & -0.042 & -0.075 \\
\hline
\end{tabular}

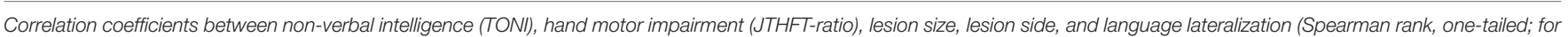

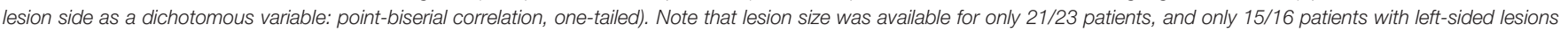
contributed data for language lateralization.

The bold values with *indicate a statistically significant correlation.

controls $(p=0.364)$ and between patients with and without epilepsy $(p=0.341$; Figure 1).

Results of the assessment of potential modifiers of nonverbal intelligence are displayed in Table 2. TONI scores were negatively correlated with the presence of epilepsy (point-biserial correlation, $r=-0.41, p<0.05$ ) and with motor impairment of the paretic hand (Spearman Rank, $r=-0.36, p<0.05$ ). Lesion size showed a trend $(r=-0.32 ; p=0.078)$ toward inferior non-verbal intelligence scores in patients with larger lesions. No significant correlations were observed for lesion side $(r=0.04$; $p=0.42)$ nor, in patients with left-sided lesions, for language lateralization $(r=0.08 ; p=0.40)$.

To investigate the differential effects of the potential modifiers epilepsy, hand motor impairment, lesion size, and lesion type, we conducted a stepwise linear multiple regression analysis in the order of the strength of the correlation coefficients. Only the factor epilepsy $\left(R^{2}=0.17\right.$, beta $=-0.410, t=-2.0, p<0.05$, one-tailed) was retained in the model as a statistically significant predictor, with epilepsy explaining $17 \%$ of the variance in nonverbal intelligence. Neither hand motor impairment $(p=-0.13$, one-tailed) nor lesion size ( $p=0.34$, one-tailed) provided additional information.

Non-verbal intelligence did not correlate with epilepsy onset $(r=-0.61, p=0.15)$, time since seizure freedom $(r=0.20, p=0.67)$, number of different antiepileptic drugs ever used $(r=0.19 ; p=0.65)$, time between first and last seizure $(r=0.20, p=0.67)$ or history of status epilepticus $(r=-0.10, p=0.85)$. 
Seven patients had right-sided lesions, 16 patients had leftsided lesions (Figure 2). In the 16 patients with left-sided lesions, functional MRI revealed left-lateralized language in $7 / 16$ patients and right-lateralized language in $8 / 16$ patients. Patient V09 could not be classified due to technical failure, and no patient showed bilateral language. Language lateralization indices ranged from +0.85 to -0.87 and correlated significantly with lesion size ( $r=-0.67, p<0.05$; Spearman rank): the larger the left-sided lesion, the stronger the right-sided language dominance.

In our healthy control group, the median in non-verbal intelligence (IQ = 103) was slightly above the population-based average (IQ = 100), but they did not differ from the populationbased estimated distribution (Chi Square $=5.590 ; p=0.232$ ). Overperformance of our healthy peer group can therefore be ruled out as the decisive factor.

\section{DISCUSSION}

The major finding of our study was that epilepsy is a key risk factor for impaired non-verbal cognitive abilities in children with perinatal stroke. This has already been known for severe pharmaco-refractory epilepsies $(3,4,21,22)$; in the current study, we could demonstrate that negative effects can also be seen even in patients with well-controlled epilepsies, i.e., with seizurefreedom for at least 6 months. Despite our comprehensive assessment, none of the other potentially modifying factors lesion size, lesion side, language lateralization, or hand motor impairment played any role beyond the deleterious effects of epilepsy.

According to our analyses, patients without epilepsies after perinatal stroke can be expected to develop normal non-verbal cognitive functions. This is in line with other studies $(3,4)$ reporting non-verbal cognitive performances in the range of typically developing children for non-epileptic patients after perinatal stroke. Furthermore, we found no correlation between TONI scores and lesion size in this group $(r=0.02)$. This confirms the report by Lõo et al., demonstrating that, in non-epileptic children with PVI, lesion size did not correlate with cognitive abilities (7). These patients provide impressive examples of the neuroplastic potential of the developing human brain: Even patients with very large lesions (e.g., patient T33) have the potential to develop non-verbal intelligence in the normal range $(\mathrm{TONI}=97)$. This neuroplastic potential basically of one hemisphere seems sufficient even in patients who shift language to the right hemisphere as a consequence of large left-sided lesions - thus apparently contradicting the "crowding hypothesis" (see below).

In contrast, in patients with epilepsies, we observed a significant impairment of non-verbal cognitive abilities, with scores ranging between 77 and 104, hence from "poor" to "average" cognitive ability according to the TONI-4-manual (28).

This is all the more striking as we had already excluded patients with pharmaco-refractory epilepsies (i.e., with ongoing seizures) and patients with a previous diagnosis of intellectual disability. Apparently, in the presence of epilepsy, the neuroplastic potential of the contra-lesional hemisphere is not sufficient to allow for an undisturbed cognitive development. Network formation must be expected to be an important step in establishing the neural substrates for higher cognitive functions. Several mechanism have been discussed how perinatal lesions lead to epileptogenesis later in life; it seems likely that epilepsy impairs neuroplasticity by interfering with network formation (42).

In order to exclude that our results are mostly driven by the one patient with seizure-freedom for $<1$ year (T13), we conducted all analyses again without this patient T13. We found that neither the results in the correlation analysis, nor the group comparisons changed after the exclusion of this patient; patients with epilepsy still scored significantly lower than controls $(p=0.007)$.

The same holds true for the multiple regression analysis; only the factor epilepsy $\left(R^{2}=0.20\right.$, beta $=-0.444, t=-2.1, p<0.05$, one-tailed) was retained in the model as a statistically significant predictor, with epilepsy explaining $20 \%$ of the variance in nonverbal intelligence.

In our cohort, none of the parameters characterizing epilepsy (i.e., epilepsy onset, time since last seizure, number of different antiepileptic drugs ever used, time between first and last seizure, or history of status epilepticus) correlated with non-verbal intelligence. This negative result must be interpreted, however, in the context of our small number of only eight patients with epilepsy and the limited dataset we obtained. We would expect that a more detailed analysis of a larger cohort of patients with stroke-induced epilepsy reveals such correlations.

Supporting our findings, impaired development of cognitive abilities in patients with epilepsies after perinatal stroke was also reported in previous studies $(3,4,7)$. Two of these studies $(3,4)$ added a longitudinal aspect to this negative influence of epilepsy, describing a decline of cognitive functions over time associated with post-neonatal epilepsy in cohorts of children with perinatal AIS. Since our study was cross-sectional in nature, we are unable to provide such insights into the time-course of the development of cognitive impairment in our patients. Furthermore, these studies $(3,4,7)$, reported on cohorts of patients with various seizure severities, ranging from seizure-free patients on anticonvulsive medication (as in most of our patients) to patients with drug-resistant epilepsies with ongoing seizures. The new aspect of our study in this respect, with seizure-freedom for at least 6 months as an inclusion criterion, was that the presence of even well-controlled epilepsy can significantly impair the development of non-verbal cognitive functions.

Nevertheless, children with larger lesions seem to be at higher risk to show impaired non-verbal cognitive abilities, since lesion size tended to correlate with non-verbal intelligence $(r=-0.32)$. Our multiple regression analysis, however, demonstrated that this correlation is not an effect of lesion size per se, but arises as a consequence of the higher likelihood of larger lesions to result in epilepsies $(r=0.55)$. Hence, the effect of lesion size is biased by the higher likelihood of epilepsy in these patients.

In addition to the fact that motor impairment can artificially influence the test procedure itself, impaired motor abilities might limit a child's abilities to explore its environment, and thereby impair also the development of cognitive functions 


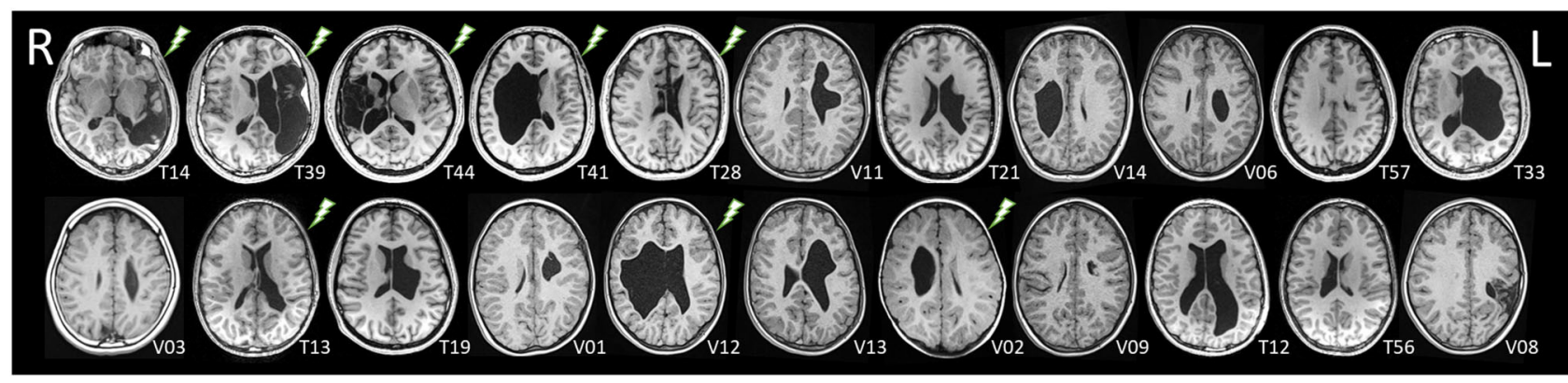

FIGURE 2 | Axial slices of the T1-weighted datasets of the 22 patients with MRI data available, sorted by TONI scores (as in Table 1). No MRI was available in patient V04 due to dental braces. A "lightning" symbol marks patients with epilepsies. Radiological orientation, R, right; L, left.

(29). Therefore, motor function is also an influencing factor when investigating cognitive outcome in children with unilateral perinatal stroke.

Similar to the lesion size analysis, children with hand motor impairment tend to show more severely impaired non-verbal cognitive abilities $(r=-0.36)$. After inclusion of epilepsy as the first regressor, however, motor impairment ended up showing no additional significant effect in the regression model. This might indicate that epilepsy and motor impairment are epiphenomena of the same underlying feature of the lesion.

An interesting discrepancy arose when comparing our findings with previous data from our own group (14) regarding the crowding hypothesis. In this previous study (14), the degree of right-hemispheric language involvement did correlate with non-verbal cognitive parameters in a similar group of patients with unilateral PVI without epilepsies. Such a correlation was not only observed for the (potentially motor-contaminated) performance IQ scores of the Wechsler Intelligence Scales, but also for motor-free tests of visuospatial memory (BlockTapping Test) and spatial ability (Tube Figures Test). To ensure that the presence of epilepsy in the current study did not wash out the influence of language lateralization or lesion size on the cognitive performances, we conducted the same analysis confined to patients without epilepsies - and again found no significant correlation between non-verbal intelligence and language lateralization $(n=12, r=-0.54, p=0.09)$ or lesion size $(n=14, r=0.02, p=0.96)$.

This discrepancy may indicate that not all non-verbal cognitive functions are compromised to the same extent: In the current study, we found no evidence for non-verbal reasoning (as measured with the TONI) to be impaired by a right-shift of language. This is compatible with previous suggestions of spatial ability and reasoning tests being separable dimensions (43). Therefore, these two studies from our group seem to indicate that language shift to the right hemisphere may compromise some originary right hemispheric functions [such as visuospatial memory and spatial ability (14)] more than others (non-verbal reasoning-the current study).

Interestingly, the factor "lesion side" was irrelevant, which is compatible with recent studies that also failed to find such a difference using subtests of the Wechsler Intelligence Tests to assess non-verbal cognitive abilities $(3,4)$. This corroborates the hypothesis that cross-hemispheric reorganization may be an important mechanism underlying the neuroplastic potential of the developing human brain (44). In contrast, two studies reported lesion side-specific problems in cognition after perinatal strokes: In the first study (12), patients with perinatally acquired right-sided lesions were impaired in configural processing and made more global errors in reproducing memorized objects in drawing tasks, whereas patients with left-sided lesions were impaired in featural progressing and made more local errors. A possible explanation for this discrepancy is, again, that side-specific problems may exist for visuospatial memory functions, but that this side-specificity can not necessarily be transferred to the context of non-verbal reasoning as measured with the TONI in the current study. Second, in an earlier study from the same group (13), rightsided lesions lead to impaired visuospatial functions in preschool children with perinatally acquired lesions. This specific impairment (which was revealed in drawing tasks) improved until school age, most likely by developing compensatory strategies. Therefore, the discrepancy to our data may not only be explained by the different cognitive functions tested, but also by the fact that we included only patients aged 8 years or older.

Limitations of our study include the following: First, we did not include patients with IQ $<70$. Therefore, we have certainly underestimated the impairment of cognitive functions caused by epilepsies. Second, we have not collected data on learning disabilities. Hence, we cannot exclude that despite average score in non-verbal cognitive abilities, our patients show deficits in these aspects of cognition. Third, we did not analyze EEG data of our patients. Therefore, we could not assess the potential influence of interictal epileptic activity on non-verbal cognitive development.

Fourth, our patients showed a wide age range. We controlled for this issue by using age-adjusted norms and controls, but the inhomogeneity of the group might still be a confounding factor. Fifth, our sample size is relatively small. Given the 
stringent inclusion criteria and the rarity of the underlying medical conditions, however, we attained an exceptional group size and used appropriate statistical approaches. We therefore interpret our results as valid. Sixth, our manual approach to determine lesion size is precise, but not at all topographically specific. We therefore cannot exclude that certain lesion topographies exert a more prominent effect on non-verbal cognitive abilities than what we report here. To this effect, lesion-symptom mapping approaches would have been necessary.

Seventh, AIS and PVI are two different pathological entities with different etiologies and different outcomes. Due to the small number of only 4 patients with AIS in our sample, we were unable to search for potential effects of lesion type.

Eighth, we did not control for the educational level of the parents-a factor which might also play a role in cognitive outcomes.

Ninth, with only two patients without CP in our cohort, we were unable to analyze the effect of presence vs. absence of $\mathrm{CP}$ on brain organization. Future studies could address this point by specifically recruiting patients without any motor impairment.

Our study has important implications for patient counseling: Patients with perinatal strokes without epilepsy can be expected to develop non-verbal intelligence within the range of typically developing children. If not hampered by epilepsy, the neuroplastic potential of one healthy hemisphere is able to support the development of normal non-verbal cognitive abilities, regardless of lesion size, lesion side, or language lateralization. Epilepsy, even when well-controlled, seems to substantially reduce this neuroplastic potential, resulting in impaired non-verbal abilities.

\section{REFERENCES}

1. Laugesaar R, Kolk A, Tomberg T, Metsvaht T, Lintrop M, Varendi H, et al. Acutely and retrospectively diagnosed perinatal stroke: a population-based study. Stroke. (2007) 38:2234-40. doi: 10.1161/STROKEAHA.107.483743

2. Fluss J, Dinomais M, Chabrier S. Perinatal stroke syndromes: similarities and diversities in aetiology, outcome and management. Eur J Paediatr Neurol. (2019) 23:368-83. doi: 10.1016/j.ejpn.2019.02.013

3. Ballantyne AO, Spilkin AM, Hesselink J, Trauner DA. Plasticity in the developing brain: intellectual, language and academic functions in children with ischaemic perinatal stroke. Brain. (2008) 131:297585. doi: 10.1093/brain/awn176

4. van Buuren LM, van der Aa NE, Dekker HC, Vermeulen RJ, van Nieuwenhuizen $\mathrm{O}$, van Schooneveld MM, et al. Cognitive outcome in childhood after unilateral perinatal brain injury. Dev Med Child Neurol. (2013) 55:934-40. doi: 10.1111/dmcn.12187

5. Greenham M, Anderson V, Mackay MT. Improving cognitive outcomes for pediatric stroke. Curr Opin Neurol. (2017) 30:12732. doi: 10.1097/WCO.0000000000000422

6. Hajek CA, Yeates KO, Anderson V, Mackay M, Greenham M, Gomes A, et al. Cognitive outcomes following arterial ischemic stroke in infants and children. J Child Neurol. (2013) 29:887-94. doi: 10.1177/0883073813491828

7. Lõo S, Ilves $\mathrm{P}$, Männamaa M, Laugesaar R, Loorits $\mathrm{D}$, Tomberg $\mathrm{T}$, et al. Long-term neurodevelopmental outcome after perinatal arterial ischemic

\section{DATA AVAILABILITY STATEMENT}

The original contributions presented in the study are included in the article/supplementary material, further inquiries can be directed to the corresponding author.

\section{ETHICS STATEMENT}

The studies involving human participants were reviewed and approved by Ethik-Kommission an der Medizinischen Fakultät der Eberhard Karls Universität und am Universitätsklinikum Tübingen (Nr. 693/2014B01). Written informed consent to participate in this study was provided by the participants' legal guardian/next of kin.

\section{AUTHOR CONTRIBUTIONS}

KL and MS conceptualized and initiated the study. AG, MH, LS, and MS collected the data. PH, MW, LS, AG, and MS conceptualized and interpreted the (f)MRI and lesion size data. AG and MS analyzed and interpreted the data and prepared the manuscript. $\mathrm{KL}, \mathrm{MH}$, LS, MW, and $\mathrm{PH}$ reviewed the manuscript. All authors approved the final manuscript as submitted.

\section{FUNDING}

This research study was supported by the German Research Foundation (DFG LI1925/4-1).

\section{ACKNOWLEDGMENTS}

$\mathrm{PH}$ was supported through a BIH Clinical Fellowship by Stiftung Charité.

stroke and periventricular venous infarction. Eur J Paediatr Neurol. (2018) 22:1006-15. doi: 10.1016/j.ejpn.2018.07.005

8. Nelson KB, Lynch JK. Stroke in newborn infants. Lancet Neurol. (2004) 3:150-8. doi: 10.1016/S1474-4422(04)00679-9

9. Dunbar M, Kirton A. Perinatal Stroke. Semin Pediatr Neurol. (2019) 32:100767. doi: 10.1016/j.spen.2019.08.003

10. Lehman LL, Rivkin MJ. Perinatal arterial ischemic stroke: presentation, risk factors, evaluation, and outcome. Pediatr Neurol. (2014) 51:7608. doi: 10.1016/j.pediatrneurol.2014.07.031

11. Akshoomoff NA, Feroleto CC, Doyle RE, Stiles J. The impact of early unilateral brain injury on perceptual organization and visual memory. Neuropsychologia. (2002) 40:539-61. doi: 10.1016/S0028-3932(01)00129-4

12. Stiles J, Stern C, Appelbaum M, Nass R, Trauner D, Hesselink J. Effects of early focal brain injury on memory for visuospatial patterns: selective deficits of global-local processing. Neuropsychology. (2008) 22:6173. doi: 10.1037/0894-4105.22.1.61

13. Stiles J, Trauner D, Engel M, Nass R. The development of drawing in children with congenital focal brain injury: evidence for limited functional recovery. Neuropsychologia. (1997) 35:299-312. doi: 10.1016/S0028-3932(96) 00088-7

14. Lidzba K, Staudt M, Wilke M, Krägeloh-Mann I. Visuospatial deficits in patients with early left-hemispheric lesions and functional reorganization of language: consequence of lesion or reorganization? Neuropsychologia. (2006) 44:1088-94. doi: 10.1016/j.neuropsychologia.2005.10.022 
15. Rasmussen T, Milner B. The role of early left-brain injury in determining lateralization of cerebral speech functions. Ann N Y Acad Sci. (1977) 299:35569. doi: 10.1111/j.1749-6632.1977.tb41921.x

16. Strauss E, Satz P, Wada J. An examination of the crowding hypothesis in epileptic patients who have undergone the carotid amytal test. Neuropsychologia. (1990) 28:1221-7. doi: 10.1016/0028-3932(90)90057-U

17. Teuber H. Why two brains? In: Schmitt FO, Worden FG, editors. Neurosciences: Third Study Program. Boston, MA: MIT Press (1974). p. 71-4.

18. Chabrier S, Peyric E, Drutel L, Deron J, Kossorotoff M, Dinomais M, et al. Multimodal outcome at 7 years of age after neonatal arterial ischemic stroke. J Pediatr. (2016) 172:156-61.e3. doi: 10.1016/j.jpeds.2016.01.069

19. Wanigasinghe J, Reid SM, Mackay MT, Reddihough DS, Harvey AS, Freeman JL. Epilepsy in hemiplegic cerebral palsy due to perinatal arterial ischaemic stroke. Dev Med Child Neurol. (2010) 52:1021-7. doi: 10.1111/j.1469-8749.2010.03699.x

20. Laugesaar R, Vaher U, Lõo S, Kolk A, Männamaa M, Talvik I, et al. Epilepsy after perinatal stroke with different vascular subtypes. Epilepsia Open. (2018) 3:193-202. doi: 10.1002/epi4.12104

21. Wagenaar $N$, Martinez-Biarge $M$, van der Aa NE, van Haastert IC, Groenendaal F, Benders $M$, et al. Neurodevelopment after perinatal arterial ischemic stroke. Pediatrics. (2018) 142:e20174164. doi: 10.1542/peds.2017-4164

22. Bajer C, Hofer W, Pieper T, Kudernatsch M, Holthausen H, Staudt M. Correlates of intellectual development before and after hemispherotomy: an analysis of 75 children and adolescents. Epileptic Disord. (2020) 22:57181. doi: 10.1684/epd.2020.1193

23. Levine SC, Kraus R, Alexander E, Suriyakham LW, Huttenlocher PR. IQ decline following early unilateral brain injury: a longitudinal study. Brain Cognit. (2005) 59:114-23. doi: 10.1016/j.bandc.2005.05.008

24. Ricci D, Mercuri E, Barnett A, Rathbone R, Cota F, Haataja L, et al. Cognitive outcome at early school age in term-born children with perinatally acquired middle cerebral artery territory infarction. Stroke. (2008) 39:40310. doi: 10.1161/STROKEAHA.107.489831

25. Westmacott R, McDonald KP, Roberts SD, deVeber G, MacGregor $\mathrm{D}$, Moharir $\mathrm{M}$, et al. Predictors of cognitive and academic outcome following childhood subcortical stroke. Dev Neuropsychol. (2018) 43:70828. doi: 10.1080/87565641.2018.1522538

26. Foo RY, Guppy M, Johnston LM. Intelligence assessments for children with cerebral palsy: a systematic review. Dev Med Child Neurol. (2013) 55:9118. doi: $10.1111 / \mathrm{dmcn} .12157$

27. Ritter N, Kilinc E, Navruz B, Bae Y. Test review. J Psychoeduc Assess. (2011) 29:484-8. doi: 10.1177/0734282911400400

28. Brown L, Sherbenou RJ, Johnsen SK. Test of Nonverbal Intelligence: TONI-4. Austin, TX: Pro-ed (2010).

29. Pavlova MA, Krägeloh-Mann I. Limitations on the developing preterm brain: impact of periventricular white matter lesions on brain connectivity and cognition. Brain. (2013) 136:998-1011. doi: 10.1093/brain/aws334

30. Raju TN, Nelson KB, Ferriero D, Lynch JK. Ischemic perinatal stroke: summary of a workshop sponsored by the National Institute of Child Health and Human Development and the National Institute of Neurological Disorders and Stroke. Pediatrics. (2007) 120:609-16. doi: 10.1542/peds.2007-0336

31. Rorden C, Karnath HO, Bonilha L. Improving lesion-symptom mapping. J Cogn Neurosci. (2007) 19:1081-8. doi: 10.1162/jocn.2007.19.7.1081

32. Wilke M, Lidzba K, Staudt M, Buchenau K, Grodd W, Krägeloh-Mann I. An fMRI task battery for assessing hemispheric language dominance in children. NeuroImage. (2006) 32:400-10. doi: 10.1016/j.neuroimage.2006.03.012
33. Meinhold T, Hofer W, Pieper T, Kudernatsch M, Staudt M. Presurgical language $\mathrm{fMRI}$ in children, adolescents and young adults. Clin Neuroradiol. (2020) 30:691-704. doi: 10.1007/s00062-019-00852-7

34. Máté A, Lidzba K, Hauser TK, Staudt M, Wilke M. A “one size fits all” approach to language fMRI: increasing specificity and applicability by adding a self-paced component. Exp Brain Res. (2016) 234:67384. doi: 10.1007/s00221-015-4473-8

35. Lidzba K, de Haan B, Wilke M, Krägeloh-Mann I, Staudt M. Lesion characteristics driving right-hemispheric language reorganization in congenital left-hemispheric brain damage. Brain Lang. (2017) 173:1-9. doi: 10.1016/j.bandl.2017.04.006

36. Jebsen RH, Taylor N, Trieschmann RB, Trotter MJ, Howard LA. An objective and standardized test of hand function. Arch Phys Med Rehabil. (1969) 50:311-9.

37. Tipton-Burton M. Jebsen-Taylor hand function test. In: Kreutzer JS, DeLuca J, Caplan B, editors. Encyclopedia of Clinical Neuropsychology. New York, NY: Springer New York (2011). p. 1365.

38. Adler C, Berweck S, Lidzba K, Becher T, Staudt M. Mirror movements in unilateral spastic cerebral palsy: specific negative impact on bimanual activities of daily living. Eur J Paediatr Neurol. (2015) 19:504-9. doi: 10.1016/j.ejpn.2015.03.007

39. Islam $M$, Nordstrand L, Holmström L, Kits A, Forssberg $H$, Eliasson AC. Is outcome of constraint-induced movement therapy in unilateral cerebral palsy dependent on corticomotor projection pattern and brain lesion characteristics? Dev Med Child Neurol. (2014) 56:252-8. doi: $10.1111 /$ dmcn. 12353

40. Sakzewski L, Ziviani J, Boyd R. The relationship between unimanual capacity and bimanual performance in children with congenital hemiplegia. Dev Med Child Neurol. (2010) 52:811-6. doi: 10.1111/j.1469-8749.2009. 03588.x

41. Reedman SE, Beagley S, Sakzewski L, Boyd RN. The Jebsen Taylor test of hand function: a Pilot test-retest reliability study in typically developing children. Phys Occup Ther Pediatr. (2016) 36:292-304. doi: 10.3109/01942638.2015.10 40576

42. Becker AJ. Review: animal models of acquired epilepsy: insights into mechanisms of human epileptogenesis. Neuropathol Appl Neurobiol. (2018) 44:112-29. doi: 10.1111/nan.12451

43. Gittler G. Sind Raumvorstellung und Reasoning separierbare Fähigkeitsdimensionen? Dimensionalitätsanalysen zweier Raschskalierter Tests: 3 DW und WMT. Diagnostica. (1999) 45:6981. doi: 10.1026//0012-1924.45.2.69

44. Krägeloh-Mann I, Lidzba K, Pavlova MA, Wilke M, Staudt M. Plasticity during early brain development is determined by ontogenetic potential. Neuropediatrics. (2017) 48:66-71. doi: 10.1055/s-0037-15 99234

Conflict of Interest: The authors declare that the research was conducted in the absence of any commercial or financial relationships that could be construed as a potential conflict of interest.

Copyright (c) 2021 Gschaidmeier, Heimgärtner, Schnaufer, Hernáiz Driever, Wilke, Lidzba and Staudt. This is an open-access article distributed under the terms of the Creative Commons Attribution License (CC BY). The use, distribution or reproduction in other forums is permitted, provided the original author(s) and the copyright owner(s) are credited and that the original publication in this journal is cited, in accordance with accepted academic practice. No use, distribution or reproduction is permitted which does not comply with these terms. 